\title{
Kampf um Öffentlichkeit. Kapitalistische Landnahme und die Zerstörung von Vernunft
}

\author{
Klaus Dörre
}

Keywords: Landnahme, Strukturwandel der Öffentlichkeit, digitalisierter Kapitalismus, öffentlich-rechtlicher Rundfunk, Journalismus, Nachhaltigkeitsziele, Mediensozialismus

\section{Abstract}

Gegenwärtig erleben wir eine kapitalistische Landnahme des Öffentlichen und der Öffentlichkeit, die auf eine Zerstörung kommunikativer Vernunft hinausläuft. So lautet die These des Beitrags, der das Ringen um Öffentlichkeit als eine neue Form des Klassenkampfs begreift. Ausgehend von einer Theorie kapitalistischer Landnahmen werden die Dynamiken einer Kommodifizierung von Wissen und Erfahrung analysiert, die einen tiefgreifenden Wandel demokratischer Öffentlichkeiten mit sich bringen. Öffentlichrechtliche Medien werden als positive Externalitäten begriffen, die es gegen den Zugriff privater Verwertungsinteressen zu verteidigen und auszubauen gilt. Mediensozialismus umschreibt die Zielkoordinaten einer solchen Politik, die Nachhaltigkeitsziele als ihre normative Grundlage nutzen kann.

Klaus Dörre: Kampf um Öffentlichkeit. Kapitalistische Landnahme und die Zerstörung von Vernunft. In: Nils S. Borchers, Selma Güney, Uwe Krüger und Kerem Schamberger (Hrsg.): Transformation der Medien - Medien der Transformation. Verhandlungen des Netzwerks Kritische Kommunikationswissenschaft. Frankfurt am Main: Westend 2021. DOI: https://doi. org/10.53291/TMQ05163.

Prof. Dr. Klaus Dörre I Friedrich-Schiller-Universität Jena I klaus.doerre@ uni-jena.de 
Wenige Tage nach dem bewaffneten Sturm auf das Capitol sperrte Twitter das private Konto des damals noch amtierenden US-Präsidenten Donald Trump. Jahrelang hatte er die mediale Öffentlichkeit mit seinen Tweets vor sich hergetrieben. Um die eigene Anhängerschaft zu erreichen, schienen ihm die einstigen Leitmedien wenn nicht überflüssig, so doch als nachrangige Teilöffentlichkeit, die allenfalls als Instrument politischer Polarisierung taugten. Über Jahre warteten Tausende Journalist*innen auf die Kurzbotschaften Trumps, stets getrieben von der Sorge, andere Medien könnten die Erstmeldung für sich reklamieren. Nach einer Rede, die rechtsradikale Demonstrant*innen zum Sturm auf das Parlamentsgebäude animiert hatte, war zunächst das Ende dieses Twitter-Regimes gekommen. Endlich, so möchte man meinen. Doch die Reaktion der deutschen Kanzlerin zeugte keineswegs von Erleichterung. Sie halte die Sperrung des privaten Twitter-Kontos von Donald Trump für problematisch, ließ Angela Merkel verlauten. Und sie hat recht! Maßt sich doch ein privates Unternehmen an, darüber zu entscheiden, wem Meinungsfreiheit zusteht und wem nicht.

Der Vorgang zeigt exemplarisch, was sich seit geraumer Zeit ereignet und nun verstärkt zum Gegenstand öffentlicher Auseinandersetzungen wird. Der Klassenkampf, so die hier vertretene These, ist auch zu einem Kampf um Öffentlichkeit geworden. Mehr noch: zu einem Kampf wider die Zerstörung der Vernunft. Er berührt die Grundfesten liberaler Demokratien und ist, aus einer emanzipatorischen Perspektive betrachtet, nur zu gewinnen, indem demokratische Öffentlichkeiten als ein nichtkapitalistisches Anderes begriffen werden, das es gegen jedwede Spielart marktgetriebener Landnahmen zu verteidigen gilt. Weit davon entfernt, den vielschichtigen Prozess der Kommodifizierung und Zerstörung von Öffentlichkeit auch nur annähernd beschreiben zu können, beschränke ich mich an dieser Stelle auf erste Überlegungen zu dieser Problematik. Ich beginne mit einigen konzeptuellen Anmerkungen zum Kampf um Öffentlichkeit (2), skizziere sodann die Grundidee des Landnahmetheorems (3) und widme mich nachfolgend der konkurrenzgetriebenen Landnahme von Wissen, Erfahrung und Öffentlichkeit (4), um abschließend Möglichkeiten zu einer demokratischen Gegenlandnahme auszuloten (5). 


\title{
2 Zerstörung von Öffentlichkeit
}

Das Phänomen eines gesellschaftlichen Kampfs um Öffentlichkeit ist alles andere als neu. Jürgen Habermas (1990 [1962]) hatte es bereits in seinem zum Klassiker gewordenen Strukturwandel der Öffentlichkeit systematisch behandelt und den Antagonisten »Proletariat « im übergreifenden Kontext seiner Theorie in gewisser Weise durch die korrigierende Kraft öffentlicher Diskurse und der in ihnen wirkenden kommunikativen Vernunft ersetzt. Wie er selbst in einem Vorwort zu einer späteren Ausgabe seines Werks einräumt, unterschätzte seine Stilisierung bürgerlicher Öffentlichkeit jedoch die Einflüsse konkurrierender Öffentlichkeiten. Solche Kommunikationskanäle entstehen durch Ausschluss aus der hegemonialen bürgerlichen Öffentlichkeit, notierte der Philosoph:

\begin{abstract}
Von »Ausschluß « kann in einem »Foucaultschen« Sinne die Rede sein, wenn es sich dabei um Gruppen handelt, deren Rolle für die Formierung einer bestimmten Öffentlichkeit konstitutiv ist. »Ausschluß « gewinnt einen anderen, weniger radikalen Sinn, wenn sich in denselben Kommunikationsstrukturen gleichzeitig mehrere Arenen bilden, wo neben der hegemonialen bürgerlichen Öffentlichkeit andere subkulturelle oder klassenspezifische Öffentlichkeiten unter eigenen, nicht ohne weiteres kompromissfähigen Prämissen auftreten. Den ersten Fall habe ich seinerzeit gar nicht berücksichtigt, den anderen Fall habe ich im Vorwort erwähnt, aber nicht behandelt. (Habermas 1990, 15)
\end{abstract}

In den Zeiten von Trump und Twitter gesellt sich ein dritter Fall hinzu. Die oft nur vermeintlich Ausgeschlossenen konstituieren über das Internet Öffentlichkeiten, die sich demokratischer Kontrolle weitgehend entziehen und so der bürgerlichen Öffentlichkeit samt ihren Leitmedien die hegemoniale Positionierung mehr und mehr streitig machen. Der Effekt ist ein mehr als zwiespältiger. Einerseits handelt es sich bei der bürgerlichen Öffentlichkeit um eine vermachtete Struktur, die Abweichungen vom Basiskonsens allenfalls in begrenztem Ausmaß gestattet, andererseits kann sie ihre normativ-regulative Funktion nur erfüllen, wenn sie für neue, vom Basiskonsens abweichende Entwicklungen in der Gesellschaft offen ist. Erst dann wirkt sie als ein Organ, das vernünftige Argumente in welch begrenztem Ausmaß auch immer zum Zuge kommen lässt.

Die Methode Trump steht indes für eine Spielart kapitalistischer Landnahmen, die nicht nur auf die Okkupation demokratischer Öf- 
fentlichkeit, sondern auf eine Zerstörung der Vernunft hinausläuft. Dies freilich in einem anderen Sinne, als der Anklang an Georg Lukács gleichnamige Schrift nahelegen könnte (dazu kritisch Bermbach und Trautmann 1987; Rücker 1976). Den »Weg Deutschlands zu Hitler auf dem Gebiet der Philosophie« lässt Lukács $(1954,6)$ bereits beim deutschen Idealismus beginnen und den Einbezug von Elementen Marx'scher Theorie in die deutsche Soziologie kritisiert er letztendlich als Legitimation eines Irrationalismus, der dem Faschismus den Weg bahnt. Ideologischer Maßstab ist ein kanonischer Marxismus, der allein nach Lukácz gegen das Virus des Irrationalen zu immunisieren vermag.

Ein derart enges Interpretationsschema ignoriert schon mit Blick auf die Vorgeschichte des deutschen Faschismus, dass das Hitler-Regime in vielerlei Hinsicht um die Zerstörung von Wissenschaftlichkeit schlechthin bemüht war. Genau das ist, sicher in anderen Formen und Ausprägungen, auch heute wieder der Fall. Trump und Twitter sind diesbezüglich nur die Spitze eines Eisbergs. Rechtspopulist*innen und -radikale ernten allerdings nur, was mit der Kommodifizierung des Öffentlichen und der Öffentlichkeit bereits vorbereitet wurde. Genauer: Die eingehegte, geschützte bürgerliche Öffentlichkeit ist zu einem Objekt kapitalistischer Landnahmen geworden, die sich in Etappen vollziehen und mit dem jüngsten Digitalisierungsschub einen Punkt erreicht haben, an dem statt von Strukturwandel eher von einer Zerstörung des Öffentlichen und der Öffentlichkeit gesprochen werden muss.

\section{Mechanismen kapitalistische Landnahme}

Beginnen wir mit dem Begriff der Landnahme, einer zentralen Kategorie für Theorien, die den Kapitalismus als expansives System analysieren und kritisieren. Ungeachtet ihrer sonstigen Heterogenität teilen entsprechende Konzepte die Annahme, dass sich kapitalistische Gesellschaften nicht ausschließlich aus sich selbst heraus reproduzieren können. Kapitalistische Dynamik beruht auf einer komplexen Innen-Außen-Bewegung. Stets beinhaltet sie die Internalisierung von Externem, die Okkupation eines nicht oder nicht vollständig kommodifizierten Äußeren. Land steht in diesem Zusammenhang nicht allein, ja nicht einmal in erster Linie für Grund und Boden. Der Begriff ist ein Synonym für Territorien, Produktionsweisen, Lebensformen und nicht zuletzt für Wissensbestände, die zuvor nicht oder nicht vollständig in den kapitalistischen Warentausch integriert waren. Sofern es kein funktio- 
nales Anderes zu entdecken gibt, das in Besitz genommen, in Wert gesetzt, kommodifiziert und profitabel genutzt werden kann, geraten Gesellschaften mit eingebautem Expansionsdrang an die Grenzen ihrer Entwicklungsfähigkeit. Sie stagnieren und zerfallen.

Die Landnahmekategorie verweist somit auf ein Expansionsparadoxon. Kapitalistische Produktionsweisen müssen sich ausweiten, um zu existieren. Dabei absorbieren und ruinieren sie allmählich, was für ihre Reproduktion benötigt wird. Je erfolgreicher die Akkumulations-, Wachstums- und Kommodifizierungsmaschine arbeitet, desto wirkungsvoller untergräbt sie die Selbstreproduktionsfähigkeit sozialer und natürlicher Ressourcen, ohne die moderne kapitalistische Gesellschaften nicht überlebensfähig sind. Fortgesetzte Landnahmen bewirken dennoch keinen automatischen Zusammenbruch des Kapitalismus. Vielmehr zwingen sie die dominanten kapitalistischen Akteure zur Kreation, Institutionalisierung und ständigen Modifikation von Selbststabilisierungsmechanismen. Hierzu gehören auf der makrogesellschaftlichen Ebene die moderne Kredit- und Finanzwirtschaft, das Innovationssystem, der Wohlfahrtsstaat, die organisierten Arbeitsbeziehungen, die Institutionen sozialer Reproduktion, die Regulationen der Naturverhältnisse, aber auch - wie wir noch sehen werden - die Schutzmechanismen für Informationen, Wissensbestände und selbst persönliche Erfahrungen. Auch die bürgerlich-demokratische Öffentlichkeit lässt sich als ein aktiv hergestelltes Außen kapitalistischer Marktvergesellschaftung begreifen. Ihre fortschreitende Kommodifizierung läuft auf die Internalisierung einer - positiven - Externalität ${ }^{1}$ hinaus. Grundsätzlich gilt: Ohne marktbegrenzende Institutionen und soziale Regeln, die den Warentausch mit Hilfe von Selbststabilisierungsmechanismen in die Gesellschaft einbetten, wäre die expansive Marktvergesellschaftung, die moderne Kapitalismen in all ihren Varianten auszeichnet, gar nicht denkbar.

Die Grundzüge des Landnahmetheorems habe ich an anderer Stelle ausführlich dargestellt (Dörre 2009; 2015). Nachfolgend beschränke ich mich deshalb auf einige Überlegungen, mit deren Hilfe sich das Konzept für die Interpretation des Strukturwandels von Öffentlichkeit

1 Die Kategorie der Externalität verwende ich hier als Synonym für das nichtkapitalistische Andere. Externalitäten entstehen nicht ausschließlich durch die - negative - Externalisierung von Kosten. Sie sind, etwa in Gestalt von NonProfit-Organisationen, in gemischten Ökonomien immer vorhanden, können neu geschaffen werden, eignen sich aber auch als Landnahme-Objekte. 
fruchtbar machen lässt. Nach Burkart Lutz $(1984,62)$, dem Nestor der deutschen Industriesoziologie, kann jeder Wachstumsschub als eine Phase je spezifischer Landnahmen durch den expandierenden industriell-marktwirtschaftlichen Teil der Volks- und Weltwirtschaft beschrieben werden. Lutz, der den Landnahmebegriff in die soziologische Debatte eingebracht hat, bezieht sich auf einen Theoriestrang, der bis zu Marx' Analyse der sogenannten ursprünglichen Akkumulation zurückreicht. Im ersten Band von Das Kapital veranschaulicht Marx (1973 [1867]), wie Staat, politischer Zwang und Akkumulation durch gewaltsame Enteignung (Bauernlegen), Raub von Kirchengütern sowie die Einhegung und private Nutzung von zuvor im Kollektivbesitz befindlichem Gemeindeland zu Geburtshelfern des Kapitalismus werden. Die Scheidung von Produzent*innen und Produktionsmitteln als zentrale Voraussetzung für die Entstehung doppelt freier Lohnarbeiter*innen und damit des Kapitalismus ist »in die Annalen der Menschheit eingeschrieben mit Zügen von Blut und Feuer« (ebd., 743).

Entscheidend ist jedoch nicht die von Marx sicher überzeichnete Gewaltträchtigkeit (Kocka 2013, 41) dieser ersten kapitalistischen Landnahme. Als weitaus wichtiger muss gelten, dass es sich bei der ursprünglichen Akkumulation von Beginn an um einen politischen, auf Staatsintervention beruhenden Prozess handelt. Weder die Veränderung der Eigentumsverhältnisse und die Expropriation des Landvolks noch die Disziplinierung der freigesetzten Arbeitskräfte für die neue Produktionsweise sind ohne Staatsintervention und außerökonomischen Zwang möglich (Marx 1973 [1867], 765). Marx vertritt allerdings die Ansicht, das politisch verfügte Zwangsmoment werde eine Episode in der Frühgeschichte des Kapitalismus bleiben. Im historischen Verlauf entstehe eine Arbeiterschaft, die »aus Erziehung, Tradition, Gewohnheit die Anforderungen jener Produktionsweise als selbstverständliche Naturgesetze anerkennt« (ebd.). Gewalt werde nur noch ausnahmsweise eingesetzt, die Arbeitenden könnten im Normalfall den Naturgesetzen der Produktion überlassen bleiben. Der stumme Zwang der ökonomischen Verhältnisse besiegele die Herrschaft des Kapitalisten über den Arbeiter.

In diesem Punkt hat sich Marx geirrt, wie Rosa Luxemburg (1975a [1913]) zu Recht kritisiert. Gravierende Strukturveränderungen des Kapitalismus vor Augen, lautet Luxemburgs zentrales Argument, dass sich die kapitalistische Dynamik als systemischer Zwang zu einer sich periodisch wiederholenden ursprünglichen Akkumulation interpretieren lässt. Erst durch Einverleibung von nichtkapitalisierter Arbeitskraft 
und Erde serwirbt das Kapital eine Expansionskraft, die ihm erlaubt, die Elemente seiner Akkumulation auszudehnen jenseits der scheinbar durch seine eigene Größe gesteckten Grenzen« (ebd., 305). Auf der sachlichen Ebene bleibe die Akkumulation des Kapitals an »nichtkapitalistische Kreise gebunden« (ebd., 306).

Obwohl sich Rosa Luxemburgs Theorie der externen Mehrwertrealisierung, mit der sie den Imperialismus ihrer Zeit erklären will, nicht halten lässt (dazu ausführlich Dörre 2018), bleiben ihre Überlegungen in mindestens dreierlei Hinsicht bedeutsam:

Erstens zeigt Luxemburg überzeugend, dass die kapitalistische Dynamik eine Doppelgestalt besitzt. Die eine Bewegung setzt sich in den Produktionsstätten des Mehrwerts, in den Fabriken, der durchkapitalisierten Landwirtschaft und auf den Warenmärkten durch. Hier reproduziert sich der Kapitalismus weitgehend auf seinen eigenen Grundlagen. Ausbeutung beruht in dieser Sphäre des kapitalistischen Warentauschs, den »inneren Märkten «, ${ }^{2}$ auf dem Äquivalenzprinzip.

Das heißt, die Lohnabhängigen werden, vermittelt über soziale Kämpfe und konjunkturelle Schwankungen, entsprechend der Grenzen entlohnt, die der historisch variable Wert ihrer Arbeitskraft setzt. Die andere Entwicklung bricht sich in Austauschbeziehungen zwischen der Kapitalakkumulation einerseits und nichtkapitalistischen Produktionsweisen, Schichten und Territorien andererseits Bahn. In diesen »äußeren«, weil nicht kapitalistischen Märkten, die es auch innerhalb nationaler Gesellschaften gibt, gilt das Prinzip des Äquivalententauschs auch in der Zirkulationssphäre allenfalls eingeschränkt. Hier herrschen Willkür, außerökonomische Disziplinierung, Dominanz, vor allem aber Überausbeutung ${ }^{3}$ und ungleicher Tausch. Landnahmen verschieben die

2 Die Unterscheidung zwischen Innen und Außen ist keine der "politischen Geographie«, sondern eine »der sozialen Ökonomie«: »Innerer Markt vom Standpunkt der kapitalistischen Produktion ist kapitalistischer Markt, ist diese Produktion selbst als Abnehmerin ihrer eigenen Produkte und Bezugsquelle ihrer eigenen Produktionselemente. Äußerer Markt ist die nichtkapitalistische soziale Umgebung, die seine Produkte absorbiert und ihm Produktionselemente und Arbeitskräfte liefert.« Daher vollziehen sich Eigentumsbildung und Freisetzungen nicht nur »auswärts«, in Kolonien und vorkapitalistischen Gesellschaften; als Proletarisierung von ländlichen und städtischen Mittelschichten, Handwerker*innen und kleinen Warenproduzent*innen finden sie ebenso im Inneren industriekapitalistischer Gesellschaften statt (Luxemburg 1975a, 311, 315).

3 Primäre Ausbeutung beruht in der Sphäre des Warentauschs auf dem Äquivalenzprinzip; das heißt die Lohnabhängigen werden, vermittelt über soziale 
Grenzen zwischen inneren kapitalistischen und äußeren nichtkapitalistischen Märkten. Es handelt sich dabei keineswegs um eine lineare Inwertsetzung von »neuem Land «. Vielmehr trägt jede Landnahme stets die Möglichkeit zu gesellschaftlicher Regression durch autoritär verfügte Disziplinierung in sich. Das ist unter anderem deshalb bedeutsam, weil der digitalisierte Kapitalismus mit seinen diversen Teilöffentlichkeiten keineswegs mit Fortschritt identifiziert werden muss.

Der beständige, systemisch unverzichtbare Stoffwechsel zwischen inneren und äußeren kapitalistischen Märkten läuft zweitens auf ein zeitlich lang gestrecktes »Zernagen« und »Assimilieren « (Luxemburg 1975a [1913], 364) nichtkapitalistischer Milieus hinaus. In diesem Prozess entstünden, so Luxemburg, die »seltsamsten Mischformen zwischen modernem Lohnsystem und primitiven Herrschaftsverhältnissen « (ebd., 312). Beispiele für solche Amalgamierungen seien das »Zerbröckeln« traditioneller Natural- und Bauernwirtschaften, etwa die »planmäßige, bewusste Vernichtung und Aufteilung des Gemeineigentums« (ebd., 328), welche die französische Kolonialpolitik in ihren arabischen Kolonien vornahm, oder die »Zwangslohnarbeit« (Luxemburg 1975b, 670), die spanische Eroberer zur Ausbeutung der indigenen Bevölkerung Lateinamerikas einführten. Landnahme bedeutet demnach, dass unterschiedliche Formen unfreier, prekärer und nur teilweise kommodifizierter Arbeit über längere historische Perioden hinweg konserviert, neu kombiniert und so als Arbeit für das Kapital genutzt werden. Es bilden sich hybride Verbindungen aus Lohnarbeit und unfreien, vorkapitalistischen oder prekären Arbeitsformen in unterschiedlich strukturierten Märkten heraus, deren »Stoffwechsel « dominanten Akteuren Extragewinne verspricht. Im Kontrast zur marxistischen Orthodoxie ihrer Zeit hat Luxemburg damit »eine völlig eigenständige Betrachtungsweise von Gesellschaftsformationen « begründet, die »im Gegensatz zu den linearen und evolutionistischen Auffassungen von >Fortschritt« (Löwy 2013, 54) steht. Diese impliziert die gleichgewichtige Anerken-

Kämpfe, in etwa entsprechend des Wertes ihrer Arbeitskraft entlohnt. In äußeren, nichtkapitalistischen Märkten gilt das Prinzip des Äquivalententauschs allenfalls eingeschränkt. Hier herrschen Disziplinierung, rassistische und sexistische Abwertung, Willkür und zum Teil offene Gewalt. Ausbeutung beruht hier auf diversen Formen eines ungleichen Tauschs; das heißt, die durchschnittlichen gültigen Standards für die Reproduktion der Arbeitskraft werden durch außerökonomischen Zwang systematisch unterboten. Weil solche Aneignungsformen auch schon vor dem Kapitalismus existiert haben, bezeichne ich sie als Varianten sekundärer Ausbeutung (vgl. Dörre 2017). 
nung einer begrenzten Pluralität an Antagonismen und Ausbeutungsverhältnissen. Ein solches Nebeneinander diverser Ausbeutungs- und Herrschaftsformen finden wir, wie sich zeigen wird, auch im zeitgenössischen Kapitalismus.

Strukturprägend für die kapitalistische Dynamik ist drittens ein sozialer Mechanismus, den der Sozialgeograph David Harvey (2014, 32) in Anlehnung an Rosa Luxemburg als Kapitalüberschuss-Absorptionsproblem bezeichnet hat. Damit ist gemeint, dass eine Ökonomie, die während einer Produktionsperiode wächst, in der nachfolgenden Periode absorptionsfähige Märkte für das zusätzliche Mehrprodukt schaffen muss, was ohne markterweiternde Investitionen nicht möglich ist. Je höher das Reichtumsniveau von Gesellschaften und je größer das Wirtschaftswachstum, desto schwerer wird es, neue Märkte für das anlagesuchende Kapital zu erschließen. Dieses Kapitalüberschuss-Absorptionsproblem ist die zentrale makroökonomische Triebkraft hinter kapitalistischen Landnahmen. Es erklärt, weshalb der Akkumulationsprozess des Kapitals »die unumschränkte Verfügungsmöglichkeit« über »alle Produktivkräfte der Erde« (Luxemburg 1975a, 312) benötigt, soweit diese in den Schranken der Mehrwertproduktion mobilisierbar sind. Und es macht vor allem deutlich, weshalb kapitalistische Dynamik immer wieder auf Marktexpansion, auf die Ausdehnung alter und die Entdeckung neuer Märkte hinausläuft. Der Kapitalismus ist demnach ein »einzigartiges System der Marktabhängigkeit«; er verallgemeinert »die Imperative des Wettbewerbs, der Akkumulation und der Profitmaximierung « und muss in einem Grade expandieren, »wie es mit keiner anderen Gesellschaftsform vergleichbar ist (Wood 2015, 115). Dieser Anforderung kann sich auch der zeitgenössische Kapitalismus nicht entziehen. Es sind allerdings besondere Märkte, in denen sich ein neuer Modus Operandi von Landnahmen zu bewähren hat und diejenigen, auf denen Wissen, Daten und Informationen vermarktet werden, sind für ihn von besonderer Bedeutung.

Kapitalistische Landnahmen, so können wir festhalten, generieren eine stets auf außerökonomischen Zwang angewiesene und mitunter gewaltträchtige Innen-Außen-Dialektik. Diese Bewegungsform ist immer mit der Absorption eines nicht marktförmigen Anderen verbunden. Landnahme bedeutet Kommodifizierung, Monetarisierung und Kommerzialisierung dieses Außen und damit zugleich dessen allmähliche Assimilation und Zerstörung. Deshalb sind Landnahmen endlich. Ist ein nichtkapitalistisches Anderes absorbiert, schwächt sich die 
expansive Dynamik ab, bis sich ein neues Anderes findet, das in Besitz genommen werden kann.

Landnahmen, die von der privaten Verfügung über Produktivkapital als dynamischem Prinzip angetrieben werden, erweiterte Reproduktion und Marktexpansion zum Ziel haben und unterschiedliche Modi von Ausbeutung und Dominanz durchsetzen, sind nicht nur umkämpft, sondern stets mit Landpreisgabe, präziser: mit Repulsionen, das heißt mit Bewegungen verbunden, die in gewisser Weise auf das Gegenteil von Kommodifizierung hinauslaufen. In der Akkumulation von bürokratisch-politischer Macht finden marktgetriebene Landnahmen ein expansives Pendant, das sich in spannungsvoller Ko-Evolution zur erweiterten Reproduktion des Kapitals, aber auch abgelöst von ökonomischer Expansion entfalten kann. ${ }^{4}$ Jede raum-zeitlich begrenzte und deshalb historisch einmalige Landnahme beruht auf einem besonderen Modus Operandi, der wiederum eine Vielzahl an Transformationsmechanismen ausbildet. Wird dieser dysfunktional, kann es zu großen Krisen von Kapitalakkumulation und gesellschaftlicher Reproduktion kommen. Derartige Ereignisse sind etwas völlig anderes als jene periodischen Ungleichgewichte, die mit dem Konjunkturzyklus verbunden sind. Sie erfassen das gesamte Ensemble gesellschaftlicher Verhältnisse und können deshalb nur überwunden werden, wenn die Beziehungen zwischen Akkumulationsregime, Regulationsweisen, Produktionsmodellen, Konsummustern, Lebensstilen und Reproduktionsformen völlig neu strukturiert werden. Es gilt, in den Worten von Burkart Lutz (1984, 62), geeignete "Strukturparameter « $\mathrm{zu}$ finden und zu institutionalisieren, die für eine gewisse Zeit die soziale Kohärenz kapitalistischer Gesellschaften sicherstellen.

\section{Landnahme von Wissen, Information und Öffentlichkeit}

Diese Grundidee gilt für die Landnahme des Sozialen, deren Modus Operandi die Dynamik des finanzialisierten Kapitalismus bestimmt hat, freilich nur in modifizierter Form. Eine solche Landnahme setzt mit ih-

4 Diesen Gedanken hat Hannah Arendt $(2006,312)$ in die Debatte eingebracht: »Durch eine unbegrenzte Akkumulation von Macht, das heißt von Gewalt, die kein Gesetz begrenzt, konnte eine unbegrenzte oder jedenfalls erst einmal unbegrenzt scheinende Akkumulation von Kapital vonstatten gehen.« 
ren komplexen Transfermechanismen und Bewährungsproben gewissermaßen an den marktbegrenzenden Institutionen und Regulationen an, die der wohlfahrtsstaatliche Kapitalismus hervorgebracht hat. Die Grundrichtung dieser Landnahme »zweiter Ordnung « habe ich an anderer Stelle ebenfalls ausführlich beschrieben (Dörre 2009; 2015), so dass auch hier eine knappe Bemerkung genügen soll. Zugespitzt formuliert, betrachten die Protagonist*innen dieser Landnahme, die finanzkapitalistischen Eliten und die ihnen funktional zugeordneten Klassenfraktionen, die Selbststabilisierungsmechanismen kapitalistischer Dynamik und die durch sie geschützten Bereiche als Verwertungsobjekte. Das gilt auch und gerade für den sozialen Raum von Wissen, persönlicher Erfahrung und die mit beiden korrespondierenden Öffentlichkeiten.

\section{Landnahme von »lebendigem Wissen« und Erfahrung}

Öffentlichkeit ist in gewisser Weise die politische Form gesellschaftlichen Bewusstseins (vgl. Gerhardt 2012). Sie beruht auf Wissen um Belange, die einer kollektiven Bearbeitung bedürfen und deshalb nach kommunikativem Austausch verlangen. Kapitalistische Landnahme bedeutet in diesem Zusammenhang, dass jenes Wissen, das in Informationen verwandelt - Öffentlichkeit konstituiert, zum Objekt privater Verwertungsinteressen wird. Dieser Prozess der Kommodifizierung von Öffentlichkeit hat lange vor der Digitalisierung begonnen; mit der Erfindung und Weiterentwicklung von Massenmedien setzt er sich in immer neuen Schüben durch. Digitale Technologie eröffnet dem markt- und gewinngetriebenen Zugriff auf solche Wissensbestände jedoch ungeahnte Möglichkeiten. Allerdings erfordert die Landnahme von Wissen besondere Transfermechanismen, von denen André Gorz (2004) einige in einem bemerkenswerten Essay kurz nach der Jahrtausendwende treffend analysiert hat. Die besondere Qualität der Landnahme von Wissen und in der Folge auch des öffentlich-rechtlichen Raums wurzelt in der Gegenwart vor allem darin, dass ihre Protagonist*innen mit Hilfe digitaler Technik Wissen und Erfahrung extrahieren, also von menschlichen Körpern und Gehirnen trennen, sie auf Universalmaschinen übertragen und gewinnbringend in die kapitalistische Warenform zwängen. Nun stellt Wissen jedoch keine Ware wie jede andere dar, sondern entsteht als ein Produkt nicht nur der Erwerbsarbeit, sondern der gesamten Lebens- 
tätigkeit von und der Interaktionen zwischen Personen. Im Unterschied zu »totem «, formalisiertem Wissen ist »lebendiges Wissen« (ebd., 45, 129) ${ }^{5}$ stets an die Person und hinsichtlich der Öffentlichkeit an den Homo publicus, an seine politischen Präferenzen, Werturteile und Weltsichten gebunden. Deshalb können sich weder Intelligenz an sich noch öffentliche Vernunft völlig »menschenfrei« entfalten. Wird das Wissen von lebendigen Körpern abgetrennt, verwissenschaftlicht und verwertet, nimmt es eine formalisierte Gestalt an. In diesem Aggregatzustand lässt es sich, ähnlich wie »tote Arbeit«, auch in digitalisierter Form als »totes Wissen« in den Kapitalkreislauf einbeziehen.

Zu den Eigenschaften von Wissen gehört aber, dass seine Produktivkraft dann am größten ist, wenn es von allen oder doch möglichst vielen genutzt werden kann. Es hat zunächst einen Wahrheitswert, der seinem - fiktiven - Warenwert vorausgesetzt ist. Um Wissen in die kapitalistische Form zu bringen, muss es künstlich verknappt und mit Eigentumstiteln versehen werden. ${ }^{6}$ In anderen Worten, wird es zu »neuem Land «, das es aus der Perspektive von Tech-Konzernen und unterstützenden Staaten zu extrahieren, zu kommodifizieren und profitabel zu nutzen gilt. Dadurch gerät Wissen zu einem der wichtigsten Objekte kapitalistischer Landnahme. Das Problem ist nur: Je besser diese Landnahme gelingt, desto stärker wird der eigentliche Gebrauchswert von Wissen, werden sein Wahrheitswert und seine Fähigkeit zur Reichtumsproduktion eingeschränkt. Die sogenannte Wissensökonomie enthält daher »im Grunde eine Negation der kapitalistischen Warenökonomie. Wenn man sie als neue Form des Kapitalismus behandelt, verschleiert man ihr Negativitätspotenzial«, denn nicht alles Wissen ist gleichwertig, und der Kapitalismus wertet und verwertet vor allem dasjenige Wissen, »dessen instrumentelles Potenzial offensichtlich oder vorhersehbar ist« (ebd., 74-75).

Formalisiertes digitales Wissen »[...] kann komplexe Interaktionen zwischen einer Vielzahl von Akteuren und Variablen organisieren und regeln. Es kann Maschinen, Anlagen und flexible Fertigungssysteme

5 Gorz (2004) grenzt das an menschliche Körper und persönliche Erfahrungen gebundene »lebendige« von formalisierbarem »toten« Wissen ab. Damit gewinnt er ein Kriterium, das ihm eine fundierte Kritik der sogenannten künstlichen Intelligenz erlaubt. Intelligenz kann es nach Gorz' Auffassung nur in ihrer Verknüpfung mit »lebendigem Wissen« geben, als rein maschinelle ist Intelligenz nicht herstellbar.

6 »Um als Ware verkäuflich und als Kapital verwertbar zu sein, muss Wissen folglich in Privateigentum verwandelt und verknappt werden.« (Gorz 2004, 11) 
entwerfen und lenken. Kurz, es kann die Rolle eines Fixkapitals spielen, indem es akkumulierte stote Arbeit der lebendigen, materiellen oder immateriellen Arbeit substituiert. Da die Grenzkosten der Software äußerst gering sind, kann sie sehr viel mehr Arbeit einsparen, als sie kostet und das in gigantischen, noch vor kurzem unvorstellbaren Ausmaßen. Das bedeutet, dass das formale Wissen unermesslich viel mehr 'Wert zerstört, als es zu schöpfen erlaubt. Anders gesagt, es erspart Unmengen von bezahlter gesellschaftlicher Arbeit und verkleinert folglich den (monetären) Tauschwert einer wachsenden Anzahl von Produkten und Dienstleistungen.« (ebd., 48, Hervorhebung im Original)

Man kann sicher darüber streiten, ob das in dieser von Gorz behaupteten Linearität zutrifft. Dagegen lässt sich etwa einwenden, dass er die Fähigkeiten kapitalistischer Akteure zur Wissensextraktion und -verwertung unterschätzt. So geht beispielsweise das Forscherteam um Andreas Boes (2015) davon aus, dass der »Informationsraum« eine wahre Fülle an möglichen neuen Geschäftsmodellen bietet, die sich für eine Revitalisierung des Kapitalismus geradezu aufdrängen. Mit »Informationsraum« ist gemeint, dass das Internet einen sozialen Handlungs- und Interaktionsraum konstituiert, der vielfältigsten Nutzungsformen offensteht. Mit seiner Hilfe bilde sich »eine lebendige globale Informations- und Kommunikationsumgebung « heraus, deren Zwecke und Veränderungsmöglichkeiten sich durch aktive Nutzung beständig veränderten und erweiterten: „Weil so geistige Tätigkeiten in neuer Qualität aneinander anschlussfähig werden, entsteht hier ein ganz neues Potenzial der Nutzung geistiger Produktivkraft.« (ebd., 85) Bisher, so sei hinzugefügt, sind die neuen Geschäftsmodelle allerdings relativ übersichtlich geblieben. Häufig verdrängen sie nur, was es in anderer Form auch schon zuvor gegeben hat.7 Ungeachtet der unterschiedlichen Einschätzungen über das Wachstumspotenzial der digitalen Ökonomie hat André Gorz den Aneignungsmodus der digitalen Landnahme von Wissen präzise rekonstruiert. Rohstoff dieser Landnahme sind nicht allein Daten und Informationen; »lebendiges Wissen « und an die Person gebundene Erfahrung bilden zunehmend die Quelle, auf die Verwertungsinteressen zugreifen. Damit wird künstlich, also mit Hilfe privat- oder staatskapitalistischer

7 »In digital capitalism capital aims to appropriate digital machines as instruments for political control, economic accumulation, and ideological manipulation«, beschreibt der Kommunikationswissenschaftler Christian Fuchs (2018, 287) eine Haupttendenz der digitalen Landnahme. 
Eigentumstitel, verknappt, was am produktivsten zu nutzen wäre, wenn Wissen als digitale Allmende allen zur Verfügung stünde.

\section{Austausch zwischen »inneren« und »äußeren« Märkten}

Was bedeutet all das nun für die bürgerliche Öffentlichkeit und die durch sie geleistete Informationsarbeit? Betrachten wir die Landnahme von Wissen, Information und Öffentlichkeit anhand der drei sozialen Mechanismen, die sich schon in Rosa Luxemburgs Akkumulationstheorie finden.

Beispiele für einen Stoffwechsel zwischen »inneren « und »äußeren« Märkten lassen sich im für die öffentliche Meinung konstitutiven Medienbereich leicht finden. Die kapitalistische Landnahme beginnt in der Bundesrepublik mit der Zulassung von privaten Radio- und Fernsehsendern neben den öffentlich-rechtlichen Anstalten Anfang der 1980er Jahre sowie einer fortschreitenden Kommerzialisierung von Zeitungen und Printmedien. Anders formuliert: Der öffentlich-rechtliche Rundfunk und seine TV-Sender werden als funktionales Anderes betrachtet, das sich dem Wettbewerb mit gewinngetriebenen Privatsendern aussetzen muss. Eigner dieser Sender sind wiederum überwiegend Konzerne, die zugleich einen erheblichen Teil der Printmedien kontrollieren.

Am weitesten fortgeschritten ist die Landnahme von Öffentlichkeit in den USA, wo der öffentlich-rechtliche Sektor allerdings eine deutlich andere Struktur aufweist als in Deutschland und anderen europäischen Staaten. Nichtkommerzielle TV-Sender und Rundfunkstationen sind gewissermaßen das Resultat von Gegenlandnahmen. Das Ende der 1960er Jahre gegründete National Public Radio (NPR) versammelt unter seinem Dach Radiostationen, die sich über eine Mischform aus staatlichen Geldern und Spenden finanzieren. Mit dem Public Broadcasting System, einem Netzwerk öffentlicher Fernsehsender, verhält es sich ähnlich. Die 1967 geschaffene Corporation of Public Broadcasting verteilt staatliche Gelder zu gleichen Teilen an beide Netzwerke (Neumann 2018).

Landnahme bedeutet in diesem schon immer von privaten Unternehmen dominierten Sektor vor allem Intensivierung von Konkurrenz und Regeländerung. Der Staat steuert vor allem über die Vergabe von Sendelizenzen; Kabelfernsehen und Streamingdienste sind dagegen kaum reguliert. Dadurch ist auch den privaten Sendern eine Konkurrenz erwachsen, die zur Minderung von Werbeeinnahmen geführt hat, 
mit einer enormen Konzentration von Medienmacht als Konsequenz. In den USA kontrollierten 1983 die 50 größten Unternehmen 90 Prozent der Medien. Gegenwärtig sind es nur noch sechs Konzerne, die über 90 Prozent dessen verfügen, was US-Bürger*innen über die Medien zu sehen, zu hören und zu lesen bekommen. 2010 erzielten diese Unternehmen - Comcast, News Corp, Disney, Viacom, Time Warner und CBS - einen Umsatz von insgesamt 275 Milliarden US-Dollar (Sanders 2017, 444); eine Summe, die davon zeugt, dass der Mediensektor zu einem hochgradig zentralisierten »inneren« kapitalistischen Markt geworden ist. ${ }^{8}$

Dieser »innere Markt« und seine Regeln bestimmen sukzessive die Themen, die Qualität der Berichterstattung, die Formate von Sendungen - kurzum: Sie entscheiden darüber, was eine Nachricht ist und was nicht, was in die Öffentlichkeit gehört und was nicht, was gesendet wird und was nicht. Selbiges geschieht selbstverständlich nicht in jenem trivialen Sinne, den Verschwörungsmythen unterstellen. Milliardär*innen, denen Zeitungen und Privatsender gehören, müssen nicht zum Telefon greifen, um Journalist*innen zu instruieren. Die Formierung der öffentlichen Meinung erfolgt über weitaus subtilere Mittel. Man kann von der Redaktionslinie einer Tageszeitung abweichen - zehn Prozent nach links, zehn Prozent nach rechts, ein Mehr an oppositionellem Geist hat keine Chance und schadet dem eigenen Renommee. Das jedenfalls glauben Qualitätsjournalist*innen auch hierzulande sicher zu wissen (vgl. Ulrich 2019).

Wo die Konzentration der Medienlandschaft weiter fortgeschritten ist als in Deutschland und anderen europäischen Staaten, sind die Folgen für das Nachrichtenwesen und die öffentliche Meinung entsprechend stärker offensichtlich. Der demokratische Sozialist Bernie Sanders hat dies am Beispiel seiner ersten Präsidentschaftswahlkampagne eindrucksvoll beschrieben. Zwei seiner Erfahrungen seien exemplarisch hervorgehoben. Als eine Art »Daumenregel« habe er gelernt, »dass eine Frage den Medienkonzernen umso unwichtiger erscheint, je mehr sie arbeitende Menschen betrifft, und dass die Aufmerksamkeit der Medien größer wird, je geringer die Relevanz für normale Leute ist« (Sanders 2017, 421). Die Hauptursache für diese Entwicklung sieht

8 Sanders bezieht sich auf Daten von https://www.freepress.net/. Der Mediensektor in den USA hat sich seither verändert, 2020 ist jedoch noch immer die Rede von sechs Konzernen, die $\$ 90 \%$ of the media outlets in America (Louise 2020) kontrollieren. 
Sanders darin, dass Politik in den medialen Öffentlichkeiten mehr und mehr zu Unterhaltung verkommt. Dazu passt, dass unter anderem allerlei Spektakel, Skandale, die Beleidigung von Gegner*innen und Intimitäten den Rang von öffentlichen Ereignissen eingeräumt bekommen.

Entertainer vom Schlage eines Donald Trump wissen, wie man einen derart umgestalteten öffentlichen Raum für eigene Zwecke ausnutzen kann:

Trump gewann die republikanischen Vorwahlen [vor seiner ersten Präsidentschaft; KD) mit Hilfe einer massiven Medienberichterstattung, die er mit seinen Tweets und scharfen persönlichen Angriffen auf seine Konkurrenten befeuerte. Bis Ende Februar hatte er nach Angaben des Economist in den Abendnachrichten zehnmal so viel Aufmerksamkeit erhalten wie der republikanische Senator Rubio aus Florida. Für die Medienkonzerne sind Beleidigungen und persönliche Attacken ein gefundenes Fressen. (ebd., 426)

Heute sehen wir, was daraus geworden ist - eine fatale Präsidentschaft, gestützt auf ein Verbiegen, Ignorieren und Umdeuten von Fakten, das an Georg Orwells 1984 erinnert. In diesem Roman indoktriniert ein Ministerium für Wahrheit die Menschen mit Hilfe einer eigenen Sprache, dem sogenannten »Neusprech «, nach dem Motto: »Krieg ist Frieden. Freiheit ist Sklaverei. Unwissenheit ist Stärke.«(Orwell 1949, 23)

So weit sind wir hierzulande noch lange nicht. Doch die Theatralisierung und Trivialisierung von Politik als Unterhaltung ist längst in Deutschland angekommen. Auch hier sieht sich der öffentliche Rundfunk dem Wettbewerb mit Privatsendern ausgesetzt, die von Werbung leben und denen sowohl die Werbeeinnahmen der öffentlich-rechtlichen Sender als auch der Rundfunkbeitrag ein Dorn im Auge sind. Nicht zufällig richten sich Attacken der radikalen Rechten und ihrer Unterstützer in bürgerlichen Parteien gegen Beitragserhöhungen; in Sachsen-Anhalt löste das Nein der christdemokratischen Regierungspartei zu einer minimalen Steigerung des Rundfunkbeitrags eine veritable Koalitionskrise aus. Gravierender ist jedoch, dass die großen Medienkonzerne, allen voran das Murdoch-Imperium, die Konzentration der Medienlandschaft auch in Deutschland weiter vorantreiben wollen. So greift der Murdoch-Konzern nach einigen Privatsendern, die sich in finanziellen Schwierigkeiten befinden. Was das im Erfolgsfall bedeuten könnte, hat Bernie Sanders unmissverständlich klargemacht: »In einer Zeit, in der Wissenschaftler immer eindringlicher vor der planetarischen Klimakrise warnen, bemüht sich Murdoch gemeinsam mit 
der fossilen Energiewirtschaft um die Widerlegung der Wissenschaft « (Sanders 2017, 439; vgl. auch Waterson 2020). Man beginnt zu ahnen, dass Wissenschaftsfeindlichkeit und Verschwörungstheorien keineswegs ausschließlich spontan entstehen. Es ist aber keineswegs allein der Murdoch-Konzern, der den Rechtspopulismus medial fördert. In Deutschland ist die Bild-Zeitung mit ihren Zusatzangeboten aus dem Hause Springer längst dabei, die Funktion einer »rechten Apo mit medialer Macht« zu erfüllen (Lucke 2021).

\section{Neukombinationen von »künstlerischer« Tätigkeit und prekärer Beschäftigung}

Wirkung können Verschwörungsmythen auch deshalb entfalten, weil die Landnahme von Wissen und Öffentlichkeit die Arbeits- und Beschäftigungsverhältnisse im Mediensektor neu strukturiert, worunter die Qualität der Berichterstattung leidet. Im journalistischen Bereich bringt diese Landnahme einen Arbeits- und Arbeitertypus hervor, dessen Wert vornehmlich auf Spezialkenntnissen und -qualifikationen beruht, die sich im Grunde nicht messen oder vergleichen lassen. Für die Spitzen in der Hierarchie von Journalist*innen, Redakteur*innen, Publizist*innen, Zeitungs-, Rundfunk- und Fernsehmacher*innen wird es immer schwerer, den Wert der Ware Arbeitskraft zu berechnen. Beschäftigte im wissensintensiven Medienbereichen agieren wie Künstler*innen oder Profifußballer*innen, in dem Sinne, dass ihre Einzigartigkeit und Besonderheit den nur schwer messbaren Wert der Arbeitskraft bestimmen.

Wenn er überhaupt irgendwo zu einer realen Figur geworden sein sollte, dann findet sich der Typus des Selbstunternehmers im Mediensektor, betraut mit der Arbeit an Öffentlichkeit und öffentlicher Meinung. Doch wie André Gorz richtig feststellt: Je mehr innerhalb eines Arbeits- und Beschäftigungsverhältnisses an das Selbst-Produzieren appelliert wird, desto stärker tendieren die Arbeitenden dazu, auch mehr sein zu wollen, als sie eigentlich sind. Journalist*innen betätigen sich als Schriftsteller*innen oder Wissenschaftler*innen und sie schreiben Bücher, um ihren eigentlichen Wert darzustellen. Werbegraphiker*innen erzeugen Kunstwerke und dergleichen mehr. Dies kann dazu führen, dass die Nebentätigkeit zur eigentlichen Quelle des Selbstbewusstseins wird: „Um einen Teil ihres Lebens dem totalen Verwertungszwang zu entziehen, messen die >Arbeiter des Immateriellen< schließlich ihren spielerischen, sportlichen, kulturellen und assoziativen Aktivitäten, bei 
denen das Sich-selbst-Produzieren ein Selbstzweck ist, eine größere Bedeutung bei als der Arbeit.« (Gorz 2004, 27)

Die Spitzen der Zunft haben mit flexiblen, befristeten Beschäftigungsverhältnissen keine Probleme. Am unteren Ende sieht das hingegen völlig anders aus. Die Arbeit an der Öffentlichkeit wird selbst bei öffentlich-rechtlichen Sendern von Journalist*innen verrichtet, die sich in Freie, feste Freie und Festangestellte ausdifferenzieren. Für einen erheblichen Teil der Journalist*innen gelten Arbeitsbedingungen, die der »Künstlerprekarität« ähneln. Einkommen und Beschäftigungsverhältnisse reichen lediglich für ein unsicheres Leben; allein das inhaltliche Interesse an der Tätigkeit bindet und integriert. Dem angesehenen Meinungsbildner in der Redaktion einer überregionalen Tageszeitung steht der freie Journalist einer Regionalzeitung gegenüber, der mit unsicherem Einkommen gemeinsam mit zwei Kolleg*innen als »Mädchen für alles« die Lokalberichterstattung eines großflächigen Landkreises besorgt. Solche Journalist*innen kann man auch jenseits ihrer eigentlichen beruflichen Tätigkeit treffen - etwa als Mitbesitzer*innen und Aushilfen in einem Kiosk, das bevorzugt Badegäste und Radwander*innen bewirtet, ${ }^{9}$ oder - weit häufiger und auch bedenklicher - als PR-Arbeiter*innen für private Auftraggeber (vgl. Obermaier und Koch 2015).

Im Journalismus markiert das regulierte, vertraglich geschützte und wohlfahrtsstaatlich eingehegte Beschäftigungsverhältnis einen Standard, der qua Enteignung oder Vorenthaltung von Sozialeigentum mit Beschäftigungsverhältnissen kombiniert wird, die sich durch Unsicherheit, Überausbeutung und ungleichen Tausch auszeichnen. Folgerichtig ist die Welt des Journalismus eine gespaltene. Die Grenzen zwischen freier Tätigkeit, wie es sie im Journalismus schon immer gab, und prekärer Beschäftigung fließen ineinander über. Auf der prekären Seite sind die Einkommen niedrig, die Arbeitsbedingungen schlecht, und auch die Reputation ist nicht sonderlich groß. Journalismus wird deshalb mehr und mehr zu einem Beruf, den man sich leisten können muss. Der Journalist Thomas Schnedler, der zu diesem Thema promoviert hat (Schnedler 2017), erläutert in einem Interview mit Deutschlandfunk Kultur:

Das ist tatsächlich eine große Gefahr, dass der Journalismus noch mehr als ohnehin schon zu so einer Art Elitenjob wird, den man sich leisten können muss, weil man entweder selber über die nötigen Mittel verfügt oder weil

9 Es handelt sich um ein reales Beispiel, das jedoch kein Ausnahmefall ist. 
man eben solche Sicherheitsgaranten und andere Unterstützer hat, die einem das dann erst ermöglichen. Und es würde schon zu einer großen Schieflage führen im Beruf, und auch dazu, dass bestimmte Wahrnehmungen und Erfahrungen gar nicht mehr in dem Berufsstand sich irgendwie wiederfinden. (Schnedler 2018)

Ob und wie sich die Prekarisierung des Journalismus auf die Qualität der Arbeit und damit auch auf das öffentliche Bewusstsein auswirkt, ist noch weitgehend unerforscht. Dass soziale Unsicherheit die Qualität der Berichterstattung negativ beeinflusst, die regulative Kraft bürgerlicher Öffentlichkeit beschädigt und so den Irrationalismus fördert, ist jedoch eine starke Hypothese, deren Untersuchung lohnenswert ist.

\section{Vom Überschuss-Absorptions- zum Verhaltensvorhersage-Problem}

Mit dem Internet wird alles besser, könnte man meinen. Schließlich bietet das Netz jedem und jeder die Möglichkeit, mit geringem Aufwand eigene Öffentlichkeiten jenseits institutionalisierter Formen zu kreieren und zu betreiben. Das demokratische Versprechen des Internets ist jedoch zum gegenwärtigen Zeitpunkt kaum mehr als ein schöner Schein. Tatsächlich treiben die Netzkonzerne die Konzentration von Datennutzung, Informationsgewinnung und -verbreitung weiter voran. Einerseits erscheinen die Zugänge niedrigschwellig. Zumindest in reichen Gesellschaften verfügen nahezu alle über die Produktionsmittel, die eine Teilhabe an der digitalen Ökonomie erlauben. Häufig genügt ein Notebook mit entsprechender Ausstattung, um geschäftsfähig zu sein. Die scheinbare Demokratisierung des Zugangs zu Produktionsmitteln korrespondiert jedoch andererseits mit einem hochgradig vermachteten Feld. In ihrem Atlas der digitalen Welt vermitteln die Autoren Martin Andree und Timo Thomsen (2020) einen Eindruck von Konzentrationsprozessen, die sich spontan keineswegs erschließen. Das hängt auch damit zusammen, dass niemand genau zu sagen vermag, wie groß der Anteil der GAFAM-Gruppe ${ }^{10}$ und vergleichbarer ITKonzerne am digitalen Aufmerksamkeitsmarkt tatsächlich ist.

10 Die GAFAM-Gruppe umfasst mit Google, Apple, Facebook und Microsoft einige der reichsten Unternehmen der Welt. Diese Konzerne verwerten die Verhaltensdaten von Milliarden Menschen. Herausgefordert werden sie gegenwärtig 
Um einen Eindruck von den Konzentrationsprozessen zu vermitteln, wählen die Autoren ein einprägsames Bild. Sie nutzen den Gini-Koeffizienten als Analogie, um das Ausmaß der Konzentration in einer Zahl auszudrücken. Ein Gini-Koeffizient von 1 würde bedeuten, dass eine Person alles besitzt; ein Wert 0 steht hingegen für maximale Gleichverteilung. Andree und Thomsen $(2020,30)$ haben nun die Verteilung der gemessenen Aufmerksamkeit auf das von ihnen erhobene Spektrum an 131000 Angeboten errechnet und kommen auf einen Wert von 0,988; das heißt ein winziges Spektrum an Angeboten zieht den Großteil der Aufmerksamkeit auf sich. Als Vergleichsmaßstab ziehen die Genannten die Vermögensverteilung heran. Würde man die Konzentration des Internet-Traffic darin übersetzen, besäßen die 500 wohlhabendsten Personen in Deutschland etwas über 85 Prozent des Vermögens, dem großen Rest blieben ganze 14,2 Prozent. Die Ungleichheit in der Aufmerksamkeitsverteilung ist damit noch weitaus größer als in der Vermögensverteilung (ebd., 19-20).

Von dieser Konzentration bekommen gewöhnliche Internetnutzer*innen in der Regel gar nichts mit. Eine große Vielfalt an Angeboten lässt den Gedanken an Konzentrationsprozesse gar nicht erst aufkommen. Damit entsteht eine Grundproblematik für jede Spielart regulatorischer Politik im Netz. Was, etwa beim Schutz von Urheberrechten, auf Unternehmen zielt, die den Traffic beherrschen, kann unter Umständen als staatlicher Eingriff in die Freiheiten der Internetnutzer*innen verstanden werden. Hinzu kommt, dass oft gar nicht klar ist, wie und auf welche Wissensbestände, Erfahrungen und Tätigkeiten zugegriffen wird. Medien, die für die bürgerlich-demokratische Öffentlichkeit lange Zeit konstitutiv waren, erwächst daraus eine existenzielle Bedrohung. Sie konkurrieren mit Suböffentlichkeiten im Netz, die sich der moralisch-regulativen Kraft bürgerlicher Öffentlichkeit weitgehend entziehen. Zugleich verlieren sie Werbeeinnahmen an die Internetkonzerne und deren Filialen. In dem Maße, wie sie sich für digitale Angebote öffnen, machen sie sich jedoch vom Wissen der Tech-Oligopole abhängig, oft, ohne mit den neuen Formaten kostendeckende Einnahmen generieren zu können. Die Großunternehmen der digitalen Ökonomie benötigen »proprietäre Märkte« (Dolata 2019, 182). ${ }^{11}$ Diese nicht frei zugänglichen Märkte entstehen, indem künst-

allenfalls durch chinesische IT-Konzerne, die ebenfalls monopolartige Organisationsformen aufweisen.

11 »Massive Konzentrationsprozesse, die Herausbildung von Winner-takes-all- 
lich verknappt wird, was eigentlich im Überfluss vorhanden ist. Unternehmen schaffen und verteidigen also mit staatlicher Unterstützung Wissens- und Technologiemonopole, die es überhaupt erst erlauben, den digitalen Kapitalismus zu einer gewinnbringenden Veranstaltung zu machen (Staab 2019).

\section{Zum Schluss: Für Mediensozialismus!}

Die großen Internetgiganten werden alles daransetzen, ihre Wissensund Marktmonopole zu erhalten - auch zu Lasten unabhängiger Medien. Doch es kommt eine weitere Gefahr hinzu, die Shoshana Zuboff (2019) in brillanter Weise analysiert hat. Sie spricht von einem aus dem Ruder gelaufenen »Überwachungskapitalismus«, der es gelernt habe, seine »historischen Bedingungen raffiniert auszubeuten und seinen Erfolg zu verteidigen« (ebd., 33). »Überwachungskapitalismus« bedeutet, dass sich die großen Internetkonzerne unsere Datenspuren im Netz als Gratisressource aneignen, um sie in Verhaltensvorhersageprodukte zu verwandeln, die sie daraufhin an Dritte verkaufen können. Die Nutzung solcher Daten für Interventionen in den US-Präsidentschaftswahlkampf zeigt, wie weit die Zerstörung demokratischer Öffentlichkeit bereits vorangeschritten ist. Der »Überwachungskapitalismus« läuft darauf hinaus, Verhaltenstendenzen nicht nur zu ermitteln, sondern erwünschtes Verhalten möglichst auch herbeizuführen - eine Dystopie, deren Realisierung bereits begonnen hat.

Fassen wir zusammen: Die Kommodifizierung von Medien bedingt, dass große gesellschaftliche Gruppen samt der sie betreffenden Themen aus der bürgerlichen Öffentlichkeit ausgeschlossen werden. Das wiederum macht die Medien, die Redaktionen und die Journalist*innen angreifbar. Nur vor diesem Hintergrund ist zu erklären, dass Teilöffentlichkeiten, die das Internet bietet, zum Katalysator einer Öffentlichkeitsrebellion geworden sind. Suböffentlichkeiten lassen die Leitmedien bei ihrer Kommunikation weitgehend außen vor. Das öffnet dem Irrationalismus Tür und Tor, weil gemeinsame Diskursstandards und -regeln in diesen Suböffentlichkeiten nicht mehr gelten. Die Inter-

Märkten und die Etablierung neuer natürlicher Quasi-Monopole, die das Web heute sowohl in ökonomischer als auch in sozialer Hinsicht prägen, sind die weithin sichtbaren Folgen dieser großflächigen Landnahme«, beschreibt Ulrich Dolata $(2019,182)$ die zurückliegende Digitalisierungsetappe. 
nalisierung des funktionalen Außen »Öffentlichkeit« in den kapitalistischen Verwertungsprozess hat eine Gegenbewegung im Netz ausgelöst, die jedoch kaum mehr als eine imaginäre Revolte ist, weil auch sie in den Bahnen kapitalistischer Landnahmen gefangen bleibt und der Zerstörung demokratischer Öffentlichkeit zusätzliche Schubkraft verleiht. Die Folgen sind in den USA zu besichtigen: Wahrheit wird zu Fake News, Wissenschaft zur Lüge, eine Wahlniederlage zum Sieg und der Sturm auf das Capitol zu einer verständlichen Reaktion vermeintlich Betrogener.

Lässt sich dieser Zerstörung von Vernunft etwas entgegensetzen? Ich weiß es nicht, aber ich hoffe es. Drei Maßnahmenbündel halte ich für zentral. Erstens benötigen wir einen digitalen Infrastruktur- und Mediensozialismus, der den Zugang zu demokratischen Öffentlichkeiten als öffentliches Gut betrachtet, das es zu schützen gilt. Die Verteidigung wissenschaftlicher Standards muss zwingender Bestandteil entsprechender Politiken sein. Wissenschaft dient der Wahrheitssuche. Sie beruht auf institutionalisiertem Selbstzweifel. Deshalb kann jede wissenschaftliche Erkenntnis potenziell mit einer wissenschaftlichen Gegenthese konfrontiert werden. Dies zu verdeutlichen ist jedoch etwas völlig anderes als die Verbreitung sogenannter alternativer Fakten. Ein öffentlich finanzierter Mediensektor muss der Wahrheitssuche Raum geben. Er darf nicht primär von Einschaltquoten und Zielgruppendiagnosen abhängen; seine Hauptinhalte müssen neben Unterhaltung und Zerstreuung seriöse Informationsangebote sein. Zweitens bedeutet dies, dass Leitmedien - allen voran der öffentlich-rechtliche Rundfunk - finanziell abgesichert, aber in ihrer Qualität verbessert und demokratisiert werden müssen. Ihre Kontrolle sollte Transformations- und Nachhaltigkeitsräten übergeben werden, die sich an den Sustainable Development Goals (SDGs) als normativer Grundlage orientieren und sicherstellen, dass die großen gesellschaftlichen Krisen und Probleme in geeigneter Weise zu Themen, Informationen und Nachrichten werden. Drittens schließlich bedarf es einer demokratischen Netz- und Medienpolitik, die Pluralismus garantiert und die Verteidigung der Meinungsfreiheit nicht privaten Unternehmen überlässt. Die Zerschlagung von Wissens- und Medienmonopolen ist dafür eine unabdingbare Voraussetzung.

Bringt man diese Überlegungen auf eine einfache Formel, dann sehen wir uns tatsächlich mit einer Entscheidungssituation konfrontiert, in der die Zurückdrängung des medial gestützten Irrationalismus und die Zerstörung von Vernunft nur noch mittels eines Übergangs zu ratio- 
naleren, nachkapitalistischen Gesellschaften möglich scheinen (Dörre und Schickert 2019; Dörre 2020). Diese Gesellschaften hätten sich des systemischen Zwangs zu fortgesetzten Landnahmen zu entledigen. Der Weg dorthin führt über den Ausbau positiver Externalitäten, zu denen an erster Stelle demokratische Öffentlichkeiten gehören.

\section{Literatur}

Andree, Martin, und Timo Thomsen. 2020. Atlas der digitalen Welt. Frankfurt am Main, New York: Campus.

Arendt, Hannah. 2006. Elemente und Ursprünge totaler Herrschaft. Antisemitismus, Imperialismus, totale Herrschaft. 11. Auflage. München: Piper.

Bermbach, Udo, und Günter Trautmann. 1987. Georg Lukács. Opladen: Westdeutscher Verlag.

Boes, Andreas, Tobias Kämpf, Barbara Langes, und Thomas Lühr. 2015. Landnahme im Informationsraum. Neukonstituierung gesellschaftlicher Arbeit in der »digitalen Gesellschaft«. WSI Mitteilungen 2: 77-85.

Dolata, Ulrich. 2019. Plattform-Regulierung. Koordination von Märkten und Kuratierung von Sozialität im Internet. Berliner Journal für Soziologie 29: 179-206.

Dörre, Klaus. 2009. Die neue Landnahme. Dynamiken und Grenzen des Finanzmarktkapitalismus. In: Soziologie - Kapitalismus - Kritik. Eine Debatte, herausgegeben von Klaus Dörre, Stephan Lessenich und Hartmut Rosa, 21-86. Frankfurt am Main: Suhrkamp.

Dörre, Klaus. 2015. Social Capitalism and Crisis: From the Internal to the External Landnahme. In: Sociology - Capitalism - Critique, herausgegeben von Klaus Dörre, Stephan Lessenich und Hartmut Rosa, 247-277. London, New York: Verso.

Dörre, Klaus. 2017. Ausbeutung und Leistungsgerechtigkeit - eine Forschungsheuristik. In: Leistung und Gerechtigkeit - Das umstrittene Versprechen des Kapitalismus, herausgegeben von Brigitte Aulenbacher, Maria Dammayr, Klaus Dörre, Wolfgang Menz, Birgit Riegraf und Harald Wolf, 174-190. Weinheim: Beltz Juventa.

Dörre, Klaus. 2018. Rosa Luxemburg, Die Akkumulation des Kapitals (1913). In: Geschichte des politischen Denkens. Das 20. Jahrhundert, herausgegeben von Manfred Brocker, 1. Auflage, 80-95. Berlin: Suhrkamp.

Dörre, Klaus. 2020. Die neue Lust am Sozialismus. Option für eine Nachhaltigkeitsrevolution? In: Sozial-ökologische Utopien. Diesseits oder jenseits von Wachstum und Kapitalismus?, herausgegeben von Benjamin Görgen und Björn Wendt, 249-272. München: Oekom.

Dörre, Klaus, und Christine Schickert (Hrsg.). 2019. Neosozialismus. Solidarität, Demokratie und Ökologie vs. Kapitalismus. München: Oekom.

Fuchs, Christian. 2018. Industry 4.0. The Digital German Ideology. TripleC: Communication, Capitalism \& Critique 16 (1): 280-289. 
Gerhardt, Volker. 2012. Öffentlichkeit. Die politische Form des Bewusstseins. München: C.H. Beck.

Gorz, André. 2004. Wissen, Wert und Kapital. Zur Kritik der Wissensökonomie. Zürich: Rotpunktverlag.

Habermas, Jürgen. 1990 [1962]. Strukturwandel der Öffentlichkeit. Neuauflage. Frankfurt am Main: Suhrkamp.

Harvey, David. 2014. Das Rätsel des Kapitals entschlüsseln. Hamburg: VSA.

Kocka, Jürgen. 2013. Geschichte des Kapitalismus. München: Beck.

Löwy, Michael. 2013. Westlicher Imperialismus gegen Urkommunismus. Eine neue Lesart von Rosa Luxemburgs ökonomischen Schriften. In: Rosa Luxemburgs »Akkumulation des Kapitals«. Die Aktualität von ökonomischer Theorie, Imperialismuserklärung und Klassenanalyse, herausgegeben von Ingo Schmidt, 53-62. Hamburg: VSA.

Louise, Nickie. 2020. These 6 corporations control 90\% of the media outlets in America. The illusion of choice and objectivity. TechStartups vom 18. September, https://techstartups.com/2020/09/18/6-corporations-control-90-mediaamerica-illusion-choice-objectivity-2020/. Zugegriffen: 5. Februar 2021.

Lucke, Albrecht von. 2021. Rechte APO mit medialer Macht. Blätter für deutsche und internationale Politik 3: 89-89.

Lukács, Georg. 1954. Die Zerstörung der Vernunft. Berlin: Aufbau.

Lutz, Burkart. 1984. Der kurze Traum immerwährender Prosperität. Frankfurt am Main, New York: Campus.

Luxemburg, Rosa. 1975a [1913]. Die Akkumulation des Kapitals. Ein Beitrag zur ökonomischen Erklärung des Imperialismus. In: Gesammelte Werke, Band 5, 5-411. Berlin: Dietz.

Luxemburg, Rosa. 1975b. Einführung in die Nationalökonomie. In: Gesammelte Werke, Band 5, 524-778. Berlin: Dietz.

Marx, Karl. 1973 [1867]. Das Kapital. Erster Band. In: Marx-Engels-Werke 23. Berlin: Dietz.

Neumann, Mario. 2018. So werden öffentliche Sender in den USA finanziert. Neue Zürcher Zeitung vom 10. Februar, https://www.nzz.ch/feuilleton/medien/oeffentliches-us-radio-helfen-sie-uns-ld.1354508. Zugegriffen: 5. Februar 2021.

Obermaier, Magdalena, und Thomas Koch. 2015. Mind the gap. Consequences of inter-role conflicts of freelance journalists with secondary employment in the field of PR. Journalism 16 (5): 615-629. DOI: 10.1177/1464884914528142.

Orwell, George. 1949. 1984. London: Secker and Warburg.

Rücker, Silvie. 1976. Irrationalismus. In: Historisches Wörterbuch der Philosophie, Band 4, herausgegeben von Joachim Ritter und Karlfried Gründer. Basel, Stuttgart: Schwabe.

Sanders, Bernie. 2017. Unsere Revolution. Berlin: Ullstein.

Schnedler, Thomas. 2017. Prekäre Arbeit im Journalismus. Dissertation. Universität Hamburg, https://ediss.sub.uni-hamburg.de/bitstream/ediss/6133/1/Dissertation.pdf. Zugegriffen: 5. Februar 2021.

Schnedler, Thomas. 2018. Prekäres Arbeiten in den Medien »Gefahr, dass Journalismus noch mehr Elitenjob wird«. Thomas Schnedler im Gespräch mit Isabelle 
Klein. Deutschlandfunk vom 11. September, https://www.deutschlandfunk.de/ prekaeres-arbeiten-in-den-medien-gefahr-dass-journalismus.2907. de.html?dram:article_id=427828. Zugegriffen: 5. Februar 2021.

Staab, Philipp. 2019. Digitaler Kapitalismus. Markt und Herrschaft in der Ökonomie der Unknappheit. Berlin: Suhrkamp.

Ulrich, Bernd. 2019. Alles wird anders. Das Zeitalter der Ökologie. Köln: Kiepenheuer \& Witsch.

Waterson, Jim. 2020. James Murdoch criticises father's news outlets for climate crisis denial. The Guardian vom 14. Januar, https://www.theguardian.com/ media/2020/jan/14/james-murdoch-criticises-fathers-news-outlets-for-climate-crisis-denial. Zugegriffen: 4. März 2021.

Wood, Ellen Meiksins. 2015. Der Ursprung des Kapitalismus. Eine Spurensuche. Hamburg: Laika.

Zuboff, Shoshana. 2019. Das Zeitalter des Überwachungskapitalismus. Frankfurt am Main, New York: Campus.

\section{Open Access}

Dieser Beitrag erscheint unter der Creative-Commons-Lizenz CC BY-ND 3.0 DE: https://creativecommons.org/licenses/by-nd/3.0/de/. 